\title{
Morbidade e mortalidade da população idosa de Florianópolis: um estudo comparativo entre homens e mulheres
}

\author{
Morbidity and mortality of the elderly population in Florianopolis city: a comparative study \\ between men and women

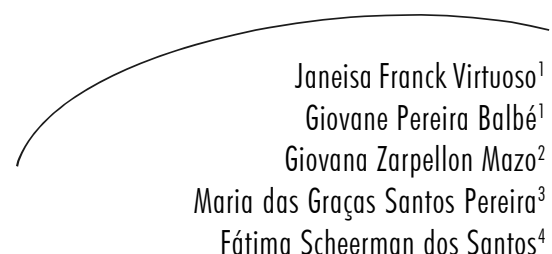

Resumo

Objetivos: Identificar e comparar as principais causas de morbi-mortalidade, entre homens e mulheres com 60 anos ou mais, residentes no município de Florianópolis - Santa Catarina, ano de 2007. Métodos: Estudo descritivo por meio de dados secundários disponíveis no Sistema de Informações de Mortalidade (SIM/SUS), do Sistema de Internações Hospitalares (SIH/SUS) e para os dados demográficos segundo o IBGE. Resultados: Identificou-se a doença isquêmica do coração, seguida da cerebrovascular e do câncer de pulmão, com os maiores coeficientes de mortalidade nos idosos. Ao comparar as principais causas de mortalidade e sexo, os homens apresentaram maior risco para câncer de pulmão, septicemia e cirrose e doença crônica do fígado (razão igual a 2,$9 ; 2,4 ; 2,4$, respectivamente). Entre as causas mais prevalentes de morbidade, destacam-se as doenças circulatórias, neoplasias e doenças respiratórias. Ao comparar as causas de morbidade ao sexo, as doenças circulatórias, seguidas das neoplasias e doenças geniturinárias, destacaram-se pela maior razão nas mulheres (razão igual a 0,93; 0,90; 0,89, respectivamente) embora com pequena diferença. Conclusão: A doença isquêmica do coração, doença cerebrovascular e câncer de pulmão foram as principais causas de óbito entre os idosos, mas comparadas ao sexo, observou-se que câncer de pulmão, septicemia e cirrose e doença crônica do fígado apresentaram-se como as principais responsáveis pela sobremortalidade masculina. As doenças circulatórias, seguidas das neoplasias e doenças respiratórias, destacaram-se pela maior causa de morbidade nos idosos, com predomínio nas mulheres, exceto as respiratórias, dando lugar às doenças geniturinárias.

\footnotetext{
Universidade do Estado de Santa Catarina. Centro de Ciências da Saúde e do Esporte. Pósgraduação em Ciências do Movimento Humano. Florianópolis, SC, Brasil.

2 Universidade do Estado de Santa Catarina, Centro de Ciências da Saúde e Esporte. . Florianópolis, SC, Brasil.

3 Universidade Federal de Santa Catarina. Graduação em Educação Física. . Florianópolis, SC, Brasil.

4 Universidade do Estado de Santa Catarina. Laboratório de Gerontologia/LAGER. Florianópolis, SC, Brasil.
}

\author{
Correspondência / Correspondence \\ Janeisa Franck Virtuoso \\ Laboratório de Gerontologia/LAGER \\ Universidade do Estado de Santa Catarina-UDESC \\ Rua Pascoal Simone, 358 - Coqueiros \\ 88080-350 - Florianópolis - SC \\ E-mail: janeisav@yahoo.com.br
}

\author{
Palavras chave: Idoso. \\ Indicadores de Morbi- \\ Mortalidade. Estudos de \\ Casos e Controles. \\ Mortalidade. Morbidade. \\ Florianópolis, SC.
}




\section{Abstract}

Objective: To identify and compare the main causes of morbi-mortality, between men and women aged 60 years or more, residents in the city of Florianópolis, Santa Catarina State, 2007. Methods: It is a descriptive study, using secondary data from the Mortality Information System (SIM/SUS), Hospital Internment System (SIH/SUS) and demographic data according to IBGE. Results: We identified ischemic heart diseases, followed by cerebrovascular and lung cancer as the highest coefficients of mortality among the elderly. Comparing the main causes of mortality and sex, men presented greater risks of lung cancer, septicemia and cirrhosis and chronic liver disease (ratios of 2,$9 ; 2,4 ; 2,4$, respectively). Among the most prevalent morbidity causes there are the circulatory diseases, cancer and respiratory diseases. Comparing the morbidity causes with sex, the circulatory diseases, followed by cancer and urinary disases presented the highest ratios among women (ratios of 0,$93 ; 0,90 ; 0,89$, respectively) although with little difference. Conclusions: Ischemic heart diseases, cerebrovascular diseases and lung cancer were the main death causes among the elderly, but when compared to sex, it was observed that lung cancer, septicemia and cirrhosis and chronic liver disease were responsible for most male deaths. The circulatory diseases, followed by cancer and respiratory diseases were the most common causes of morbidity in the elderly, mainly among women, except for respiratory diseases, replaced by urinary diseases.
Key words: Aged. Indicators of Morbidity and Mortality. CaseControl Studies. Mortality. Morbidity. Florianópolis city.

\section{INTRODUÇÃO}

O crescimento, em números absolutos e relativos, de pessoas com 60 anos de idade ou mais, é um fenômeno mundial. $\mathrm{O}$ envelhecimento populacional foi inicialmente observado em países desenvolvidos, mas recentemente é nos países em desenvolvimento que a população idosa tem aumentado de forma acentuada. ${ }^{1}$

A feminização da velhice é uma manifestação do processo de transição de gênero que acompanha o envelhecimento populacional em curso em todo o mundo, associada à maior longevidade das mulheres em comparação com os homens, bem como à maior presença relativa de mulheres na população idosa, principalmente nos estratos mais velhos. ${ }^{2}$ Diante dessa realidade, surge a importância de estudar a saúde do homem idoso, a fim de melhor entender as causas de morbi-mortalidade nesse grupo e propor uma nova gestão da velhice para a sociedade.

No Brasil, as estatísticas de mortalidade vêm sendo cada vez mais usadas como subsídio para os indicadores de saúde, visto sua eficiência e o fácil acesso. ${ }^{3,4}$ Estes indicadores têm mostrado um diferencial de mortalidade e morbidade entre homens e mulheres, ${ }^{5}$ em que a sobremortalidade masculina acontece em praticamente todas as idades e para quase a totalidade das causas, ${ }^{6,7}$ resultando na feminização da velhice.

Apesar da menor sobrevida masculina, ${ }^{7}$ quase sempre aceita sem muita discussão e vista como resultado somente de fatores biológicos, surge a necessidade de melhor entender as causas de morbi-mortalidade que acometem o homem idoso comparado ao sexo feminino, buscando-se analisar a sobremortalidade desse grupo, que carece de estudos. ${ }^{6}$

Objetivos: Identificar e comparar as principais causas de morbi-mortalidade, entre homens e mulheres com 60 anos ou mais, residentes no município de Florianópolis, Santa Catarina, com ênfase no ano de 2007.

\section{MÉTODOS}

Trata-se de estudo descritivo que caracteriza o perfil epidemiológico de morbi-mortalidade da população idosa masculina e feminina (60 anos ou mais) de Florianópolis - SC, por meio de dados secundários. 
Os bancos de dados utilizados foram o Sistema de Informações de Mortalidade (SIM/SUS), composto de informações provenientes das declarações de óbito, e o Sistema de Internações Hospitalares (SIH/SUS), construído com os dados que compõem a Autorização de Internação Hospitalar (AIH). Esses bancos estão disponíveis no site do Ministério da Saúde (DATASUS). ${ }^{8}$

Neste estudo, foram considerados óbitos e internações segundo o local de residência, para indivíduos com 60 anos ou mais, referentes a 2007. Para classificação da causa básica e específica de óbito e internação, utilizou-se a Classificação Internacional de Doenças - 10 ${ }^{a}$ Revisão (CID-10). ${ }^{9}$

Os coeficientes de mortalidade foram tratados dividindo-se o número de óbitos por causa, em pessoas com 60 anos ou mais, segundo o sexo e o número de habitantes na mesma faixa etária, multiplicado por 10 n. O número de internações foi tratado por meio de valores absolutos.

As informações sobre a população de Florianópolis segundo sexo foram obtidas por meio de estimativas populacionais do Instituto Brasileiro de Geografia e Estatística (IBGE), ${ }^{10}$ provenientes do Censo Nacional de 2000.

\section{RESULTADOS}

Segundo estimativa populacional do IBGE para 2007, a população idosa de Florianópolis alcançou o patamar de 35.041 idosos, aumento de 38,58\% comparado a 1996, sendo 14.566 homens (aumento de 38,79\%) e 20.475 mulheres (aumento de 38,43\%) em relação ao mesmo período. ${ }^{10}$
Embora haja aumento substancial no número de idosos em Florianópolis, observa-se a disparidade entre os sexos masculino e feminino, sendo os homens em menor número, fato que corrobora a feminização da velhice.

Para melhor entender esse processo, buscouse identificar e comparar as principais causas de mortalidade entre sexos, observando a série histórica nos anos de 1996, 2000, 2004 e 2007, com ênfase neste último ano (tabela 1). As três principais causas de mortalidade que apresentaram os maiores coeficientes foram as doenças isquêmicas do coração ( 92,7 para homens e 47,4 para mulheres), seguidas das doenças cerebrovasculares (28,1 para homens e 30,3 para mulheres) e câncer de pulmão ( 24,7 homens e 6,3 mulheres).

Assim, ao comparar os coeficientes das principais causas de mortalidade entre os sexos (tabela 1), o câncer de pulmão (razão igual a $3,9)$, seguido de septicemia e cirrose e doença crônica do fígado (ambas com razão igual a $3,4)$ foram as principais causas $\mathrm{da}$ sobremortalidade masculina. Outro dado importante foi a sobremortalidade masculina de quase o dobro para a doença isquêmica do coração (razão igual a 1,9) e os acidentes de transporte (razão igual a 1,8). Cabe salientar os elevados coeficientes de mortalidade segundo causas especificas entre os sexos, em que os homens apresentaram elevados valores no câncer de estômago $(13,0)$ e próstata $(11,7)$, quando comparados aos cânceres específicos da população feminina (câncer de mama - 8,8 e colo de útero - 1,5). 
Tabela 1 - Coeficientes de mortalidade (por 10.000 habitantes com 60 anos ou mais) por homens e mulheres segundo causas de óbito. Florianópolis, 1996/2007.

\begin{tabular}{|c|c|c|c|c|c|c|c|c|}
\hline \multirow[t]{2}{*}{ Causas de Óbito } & \multicolumn{2}{|c|}{1996} & \multicolumn{2}{|c|}{2000} & \multicolumn{2}{|c|}{2004} & \multicolumn{2}{|c|}{2007} \\
\hline & Homens & Mulheres & Homens & Mulheres & Homens & Mulheres & Homens & Mulheres \\
\hline Doença Isquêmica do Coração & 93,1 & 48,4 & 69,3 & 42,2 & 80,2 & 47,4 & 92,7 & 47,4 \\
\hline Doença Cerebrovascular & 41,5 & 48,4 & 42,6 & 41,0 & 32,5 & 41,5 & 28,1 & 30,3 \\
\hline Câncer de pulmão & 30,3 & 4,0 & 24,2 & 7,7 & 22,7 & 8,1 & 24,7 & 6,3 \\
\hline Bronquite, enfisema e asma & 61,7 & 34,9 & 40,1 & 24,9 & 26,5 & 22,1 & 19,9 & 11,2 \\
\hline Diabetes & 21,3 & 25,4 & 23,4 & 23,8 & 19,7 & 11,3 & 12,4 & 16,1 \\
\hline Pneumonias & 23,6 & 24,6 & 7,5 & 12,5 & 18,2 & 14,0 & 10,3 & 14,7 \\
\hline Câncer de Estômago & 15,7 & - & 9,2 & - & 9,1 & - & 13,0 & - \\
\hline Câncer de Próstata & 11,2 & - & 18,4 & - & 14,4 & - & 11,7 & - \\
\hline Câncer de mama & - & 7,1 & - & 8,3 & - & 7,0 & - & 8,8 \\
\hline Outros acidentes & 3,4 & 2,4 & 3,3 & 4,8 & 2,3 & 0,5 & 5,5 & 5,4 \\
\hline Acidente de transporte & 4,5 & 1,6 & 2,5 & 3,6 & 6,1 & 1,1 & 5,5 & 2,9 \\
\hline Insuficiência Renal & 2,2 & 4,8 & 3,3 & 2,4 & 3,8 & 1,1 & 4,8 & 2,9 \\
\hline Mal Definidas & 7,9 & 4,8 & 4,2 & 5,3 & 5,3 & 3,8 & 4,1 & 2,4 \\
\hline Septicemia & 3,7 & 7,9 & 2,8 & - & 5,0 & 8,9 & 3,8 & 1,1 \\
\hline Cirrose e doença crônica do fígado & 3,4 & - & 4,2 & 0,6 & 6,1 & 0,5 & 3,4 & 1,0 \\
\hline Câncer de colo de útero & - & 4,0 & - & 3,6 & - & 1,1 & - & 1,5 \\
\hline Infecções Intestinais & 1,1 & 1,6 & 0,8 & - & - & 1,1 & 1,4 & 1,5 \\
\hline Total & 484,5 & 343,5 & 386,5 & 308,2 & 430,6 & 306,9 & 392,0 & 292,1 \\
\hline
\end{tabular}

Verifica-se que os coeficientes específicos de mortalidade entre os sexos para as doenças supracitadas se apresentaram com pequenas variações, conforme ilustra a série histórica entre os períodos (a partir de 1996) disponíveis no DATASUS (tabela 1). Contudo, cabe destacar a bronquite, enfisema e asma como as doenças com declínio acentuado no período de 1996 a 2007, em ambos os sexos.

Quanto ao perfil de morbidade, identificou-se, por meio da distribuição das internações hospitalares segundo grupos de causas, a prevalência das doenças do aparelho circulatório, seguida das neoplasias e doenças do aparelho respiratório (tabela 2).

Tabela 2 - Frequencia (f) de internações hospitalares no SUS por homens e mulheres segundo principais grupos de causas de morbidade. Florianópolis, 1998/2007.

\begin{tabular}{|c|c|c|c|c|c|c|c|c|}
\hline \multirow[t]{2}{*}{ Principais Grupos de Causas } & \multicolumn{2}{|c|}{1998} & \multicolumn{2}{|c|}{2001} & \multicolumn{2}{|c|}{2004} & \multicolumn{2}{|c|}{2007} \\
\hline & $\begin{array}{c}\text { Homens } \\
(f)\end{array}$ & $\begin{array}{c}\text { Mulheres } \\
(f)\end{array}$ & $\begin{array}{c}\text { Homens } \\
(f)\end{array}$ & $\begin{array}{c}\text { Mulheres } \\
(f)\end{array}$ & $\begin{array}{l}\text { Homens } \\
(f)\end{array}$ & $\begin{array}{c}\text { Mulheres } \\
(f)\end{array}$ & $\begin{array}{l}\text { Homens } \\
(f)\end{array}$ & $\begin{array}{c}\text { Mulheres } \\
(f)\end{array}$ \\
\hline Doenças do aparelho circulatório & 288 & 251 & 404 & 369 & 567 & 545 & 547 & 583 \\
\hline Neoplasias (tumores) & 161 & 148 & 179 & 180 & 237 & 265 & 303 & 333 \\
\hline Doenças do aparelho respiratório & 212 & 201 & 242 & 205 & 263 & 259 & 237 & 293 \\
\hline Doenças do aparelho digestivo & 169 & 136 & 203 & 131 & 212 & 177 & 173 & 201 \\
\hline Demais causas & 261 & 270 & 257 & 256 & 195 & 180 & 154 & 184 \\
\hline Lesões envenenamento e consequentes & 32 & 67 & 34 & 60 & 73 & 95 & 76 & 113 \\
\hline Doenças do aparelho geniturinário & 73 & 70 & 85 & 74 & 91 & 102 & 97 & 108 \\
\hline Algumas doenças infecciosas e parasitárias & 18 & 23 & 54 & 47 & 49 & 81 & 71 & 79 \\
\hline $\begin{array}{l}\text { Doenças sistêmicas osteomusculares } \\
\text { e tecido conjuntivo }\end{array}$ & 16 & 22 & 23 & 55 & 34 & 80 & 36 & 57 \\
\hline $\begin{array}{l}\text { Doenças endócrinas nutricionais } \\
\text { e metabólicas }\end{array}$ & 42 & 53 & 31 & 79 & 36 & 58 & 33 & 52 \\
\hline Total & 1272 & 1241 & 1512 & 1456 & 1757 & 1842 & 1727 & 2003 \\
\hline
\end{tabular}


Entre os principais grupos de causas de internações, ao comparar os sexos no ano de 2007, constataram-se as doenças do aparelho circulatório (razão igual a 0,93), seguidas de neoplasias (razão igual a 0,90 ) e doenças do aparelho geniturinário (razão igual a 0,89), todas com razão maior para o sexo feminino.

Os maiores casos de internações nas mulheres não foram observados somente em 2007 , pois, como mostra a tabela 2, ao calcular o total de internações e comparar entre 1998 e 2007, observou-se o aumento total de internações de $110 \%$ para as doenças do aparelho circulatório (132\% no sexo feminino e $90 \%$ no masculino), seguido de $106 \%$ nas neoplasias $(125 \%$ nas mulheres e $88 \%$ nos homens) e com $43 \%$ para as doenças do aparelho geniturinário (54\% mulheres e $33 \%$ homens), evidenciando um crescente número de internações em ambos os sexos, embora com predomínio das mulheres.

\section{DISCUSSÃO}

Segundo a publicação do Ministério da Saúde e Secretaria de Vigilância em Saúde intitulada Perfil de Mortalidade do Brasileiro, ${ }^{11}$ as principais causas de morte na população com 60 anos ou mais são as doenças do aparelho circulatório, seguidas das neoplasias. Esses dados reforçam os resultados encontrados em nosso estudo, segundo o qual as doenças circulatórias (isquêmicas do coração e cerebrovasculares) e o câncer de pulmão se destacaram pela maior prevalência entre as principais causas de mortalidade na população idosa de Florianópolis em 2007. Segundo o IBGE, ${ }^{12}$ as doenças do aparelho circulatório, em 2005 , representaram $36,5 \%$ dos óbitos da população idosa no Brasil, destacando-se a Região Centro-Oeste, com 39,1\%, e a Sul, com 38,4\%. Apesar do elevado percentual para a Região Sul, observou-se em nosso estudo uma pequena redução nos coeficientes das doenças circulatórias (isquêmica e cerebrovascular) no período de 1996 a 2007, o que pode estar em parte associado à melhoria no acesso aos serviços de saúde e/ou maior conscientização, por parte da população, quanto a alimentação e prática de exercícios físicos. ${ }^{12}$ Entre outros fatores que podem estar relacionados às maiores causas de óbito por doenças circulatórias, encontram-se os modificáveis, tais como sedentarismo, tabagismo, obesidade, dislipidemia, hipertensão e diabetes. ${ }^{13}$ Embora tenham ocorrido investimentos na prevenção, observa-se a necessidade de políticas públicas que visem a criar programas de promoção de saúde, além de diagnósticos e tratamentos adequados que possam minimizar a ocorrência dos fatores supracitados, evitando o surgimento de determinadas patologias, principalmente a hipertensão e diabetes.

Ao comparar as causas de mortalidade entre os sexos, observou-se a sobremortalidade masculina decorrente do câncer de pulmão $(2,9$ vezes maior), seguida da septicemia e cirrose e doenças crônicas do fígado (ambas 2,4 vezes maior). Esse achado demonstra que a sobremortalidade dos homens idosos não necessariamente se encontra nas doenças mais prevalentes de mortalidade, mas nas decorrentes de fatores comportamentais, ligados ao gênero. Corroborando esta hipótese, acredita-se que a sobremortalidade masculina oriunda do câncer de pulmão e cirrose e doenças crônicas do fígado seja uma diferença de gênero, já que o hábito de fumar e beber faz parte de um estereótipo masculino. ${ }^{14}$ Além disso, a ocorrência tende a acontecer em idades mais avançadas, quando há um desfecho das referidas patologias, aliadas a mudanças de metabolismo que acompanham o processo de envelhecimento. ${ }^{15}$ Segundo a Organização Panamericana da Saúde, ${ }^{16}$ um terço dos casos de câncer poderia ser evitado se fossem tomadas medidas de prevenção como modificar hábitos alimentares, praticar exercícios físicos regularmente, evitar o fumo e o álcool, entre outras.

Por outro lado, quanto aos elevados coeficientes de morte por septicemia, acredita-se também estarem associados ao comportamento do sexo masculino, devido à falta de sensibilização do homem quanto a exames preventivos e procura ao atendimento médico. Essa imprudência 
masculina pode estar atrelada ao fato de só se buscar auxílio especializado após apresentar sintomas graves, o que provavelmente vem favorecer a disseminação da doença, levando ao comprometimento de vários órgãos e, consequentemente otimizando o desencadeamento de infecções pós-internação. Outra hipótese, mais contundente, é destacada por Laurenti et al (2008), ${ }^{4}$ ao realizarem estudo sobre a fidedignidade das causas de morte declaradas pelos médicos nos atestados de óbito. Os autores constataram que, apesar de existirem excelentes prontuários, a declaração do médico no atestado de óbito nem sempre corresponde aos diagnósticos neles registrados, sendo que não raro aparecem apenas "parada cardíaca", "insuficiência cardíaca" e, muito frequentemente, septicemia, sem informar a causa básica de morte.

Pode-se também considerar a maior mortalidade masculina por causas externas como uma diferença de gênero, pois o ambiente social onde o homem vive normalmente é mais estressante e mais exposto a acidentes (trabalho ou trânsito), se comparado ao ambiente doméstico, em que a maioria das mulheres vive. ${ }^{5,17,18}$ Este fato pode ser mais bem compreendido, já que, segundo Néri, ${ }^{2}$ apesar do advento da aposentadoria, atualmente $36 \%$ dos homens idosos permanecem economicamente ativos, tornando-se sujeitos a esses tipos de acidentes.

Quando se busca entender a gênese $d a$ sobremortalidade masculina por câncer de próstata e estômago, a falta de políticas públicas específicas para a saúde do homem e a baixa utilização de serviços de saúde por parte destes parecem ser fortes indícios, ${ }^{5,19,20}$ haja vista seu elevado coeficiente de mortalidade nas causas específicas, relacionado a seu par. O Ministério da Saúde, via Programa de Saúde da Família (PSF), conseguiu atingir a Saúde da Mulher com programas específicos para controle e prevenção do câncer de colo de útero e mama. Os altos coeficientes de mortalidade por neoplasias exclusivas ou predominantes no homem justificam os esforços atuais para se implantar os princípios e diretrizes da Política Nacional de Atenção Integral à Saúde do Homem. ${ }^{21}$ Essa política poderá, futuramente, diminuir os coeficientes de mortalidade encontrados.

Vale a pena acrescentar que, por uma questão cultural, os homens não se mostram sensíveis a campanhas preventivas e educativas relacionadas ao cuidado com a saúde, ${ }^{5,22}$ especialmente na prevenção do câncer de próstata. ${ }^{18}$ Mais uma vez, observa-se a necessidade de investimentos na prevenção de doenças por meio de programas que ampliem o campo de ação da medicina preventiva através de hábitos saudáveis, os quais contribuirão na redução dos diversos fatores de risco, em especial alcoolismo, tabagismo e sedentarismo.

Portanto, destaca-se a necessidade de medidas preventivas nas diversas faixas etárias, tais como na adolescência e vida adulta, para que haja uma conscientização da população quanto à mudança nos aspectos comportamentais, em especial no homem. Esta reflexão se faz necessária, pois como mostra nosso estudo, as três principais causas (câncer de pulmão, septicemia e cirrose e doença crônica do fígado) da sobremortalidade masculina nos idosos de Florianópolis, em 2007, podem estar relacionadas ao comportamento dos mesmos. Todavia, para que se possa reverter esse quadro, acreditamos ser necessário redirecionar o foco do atendimento à saúde, não ao tratamento, e sim à prevenção, maximizando a conscientização da população em faixas etárias menores. $\mathrm{O}$ Ministério da Saúde, ${ }^{23}$ conforme informações colidas pelo VIGITEL em 2007, destaca que, no Brasil, o câncer representa a segunda causa de óbito na população adulta, ficando atrás apenas das doenças cardiovasculares.

Essas colocações se refletem nos dados apresentados na série histórica (tabela 1) das principais causas da sobremortalidade masculina, onde se observa a manutenção, com mínima redução das mortes causadas por neoplasias (câncer de pulmão e cirrose doença crônica do f'́gado) entre 1996 e 2007, reforçando a hipótese de deficiência nas campanhas e programas de prevenção. 
Quanto às causas de morbidade, avaliadas neste estudo segundo as internações hospitalares, podese observar semelhança na distribuição entre sexos, segundo as principais causas (CID-10). ${ }^{9} \mathrm{~A}$ semelhança entre homens e mulheres também foi encontrada nos estudos de Laurenti et al. ${ }^{6}$ Acredita-se que, ao retirar as internações decorrentes de gestação, parto e puerpério, esses valores tendem a ser semelhantes entre as duas populações em qualquer faixa etária. Entretanto, ao se comparar as principais causas de internações entre os sexos, observou-se razão maior para o sexo feminino, quanto às doenças do aparelho circulatório, neoplasias e doenças do aparelho geniturinário.

Tal achado pode ser em parte justificado, já que as mulheres apresentam maior índice de incapacidades com o advento da idade ${ }^{24} \mathrm{e}$, consequentemente, sua probabilidade de internação passa a ser maior em relação aos homens idosos, o que facilita o diagnóstico precoce e o possível tratamento. Corroborando esta hipótese, Pinheiro et al., ${ }^{19}$ ao analisarem o perfil de morbidade referida, acesso e uso de serviços de saúde em homens e mulheres no Brasil segundo o PNAD/1998, constataram que as mulheres buscam mais serviços para realização de exames de rotina e prevenção $(40,3 \%$ mulheres e $28,4 \%$ homens), enquanto os homens procuram serviços de saúde sobretudo por motivo de doença (36,3\% homens e $33,4 \%$ mulheres).

Contudo, apesar de as mulheres serem mais conscientes na prevenção, existem agravantes que as tornam mais suscetíveis a internações, em parte devido a doenças circulatórias. Entre elas, a hipertensão e diabetes destacam-se pela maior incidência nesta população. Dados do VIGITEL/ $2007^{23}$, ao analisarem os indivíduos com 65 anos ou mais que autorreferiam diagnóstico de hipertensão arterial no Brasil, observaram que $49,4 \%$ dos homens e $62,8 \%$ das mulheres possuem diagnóstico da mesma, e 17,6\% dos homens e $19,3 \%$ das mulheres, diabetes. A hipertensão arterial é uma comorbidade particularmente comum no diabético, representando risco adicional para o desenvolvimento de complicações cardiovasculares. ${ }^{25,26}$ Dessa forma, levanta-se a hipótese de que a causa de internação por doenças circulatórias, com predomínio nas mulheres, possa ser um agravante decorrente da maior incidência de doenças como diabetes e hipertensão. Dentre as causas de internação devido às neoplasias, podem estar atreladas questões comportamentais, conforme destacado anteriormente. As causas de doenças geniturinárias podem estar relacionadas à maior procura por parte do sexo feminino dos serviços voltados à prevenção das mesmas.

No entanto, Matijascic \& Dias ${ }^{27}$ citam a necessidade de melhorias nas condições de trabalho, transporte, moradia, melhoria na qualidade da educação das famílias e eficientes políticas públicas de saúde preventiva para se garantir um envelhecimento saudável da população, tanto feminina quanto masculina.

Conclui-se, assim, que as doenças cerebrovasculares, doenças isquêmicas do coração e câncer de pulmão foram as principais causas de mortalidade nos idosos de Florianópolis em 2007. Entre as responsáveis pela sobremortalidade masculina em relação ao sexo, destacam-se o câncer de pulmão, septicemia e cirrose e doenças crônicas do fígado. Quanto às principais causas de morbidade identificadas, destaque é dado às doenças do aparelho respiratório e circulatório e neoplasias. Já se compararadas ao sexo, as duas últimas também se apresentaram responsáveis pela maior incidência de internações no sexo feminino, além das doenças geniturinárias.

Entretanto, salienta-se que os resultados encontrados devem ser interpretados com certa cautela, haja vista que se originam de informações secundárias. Estas são baseadas em prontuários, que podem apresentar falhas de preenchimento; e os bancos de dados disponíveis no DATASUS não estão livres de falhas na tabulação e atualização, o que pode ser considerado um fator limitador deste estudo.

Diante dessa realidade, intervenções mais efetivas devem ocorrer a partir de políticas públicas que estimulem a adesão dos homens ao cuidado com sua saúde, com medidas preventivas nas faixas etárias mais jovens. Sugere-se, ainda, a 
realização de novos estudos que visem a acompanhar as possíveis mudanças no perfil de morbimortalidade da população idosa, por meio dos dados disponíveis no próprio DATASUS, a fim de apontar para o direcionamento de políticas públicas mais eficazes e eficientes na prevenção.

\section{REFERÊNCIAS}

1. Mathias TAF, Mello Jorge MHP, Laurenti R. Doenças cardiovasculares na população idosa: análise do comportamento da mortalidade em município da região Sul do Brasil no período de 1979 a 1998. Arq Bras Cardiol 2004; 2(6):533-41.

2. Néri AL. Idosos no Brasil vivências, desafios e expectativas na terceira idade. São Paulo: Fundação Perseu Abramo; 2007.

3. Mello Jorge MHP, Gotlieb SLD, Laurenti R. O sistema de informações sobre mortalidade: problemas e propostas para o seu enfrentamento I - mortes por causas naturais. Rev Bras Epidemiol 2002; 5(2):197-211.

4. Laurenti R, Mello Jorge MHP, Gotlieb SLD. Mortalidade segundo causas: considerações sobre a fidedignidade dos dados. Rev. Panam. Salud. Pública/Pan. Am J Public Health 2008; 23(5): 349-56.

5. Schraiber LB, Gomes R, Couto MT. Homens na pauta da saúde coletiva. Ciên Saúde Colet 2005; 10:7-17.

6. Laurenti R, Mello Jorge MHP, Gotlieb SLD. Perfil epidemiológico da morbi-mortalidade masculina. Ciên Saúde Colet 2005; 10(1):35-46.

7. Figueiredo W. Assistência à saúde dos homens: um desafio para os serviços de atenção primária. Ciên Saúde Colet 2005; 10(1):105-9.

8. DATASUS. Banco de dados do Sistema Único de Saúde. [Disponível em: www.datasus.gov.br].

9. Organização Mundial de Saúde. Classificação estatística internacional de doenças e problemas relacionados à saúde $-10^{\mathrm{a}}$ Revisão. Centro Brasileiro de Classificação de Doenças, Edusp: São Paulo, 1995.

10. IBGE.. [on-line] 2008. [Disponível em: http:// www.ibge.gov.br. Acesso em: 25 out. 2008].

11. Brasil. Ministério da Saúde. Perfil de Mortalidade do Brasileiro. Secretaria de Vigilância em Saúde. Brasília; 2008.

12. IBGE. Indicadores Sociodemográficos e de Saúde no Brasil. Estudos e pesquisas. Informação demográfica e socioeconômica número 25. Rio de Janeiro; 2009.
13. Desai MM, Zhang P. Surveillance for morbidity and mortality among older adults - United States, 1995-1996. Morbidity and Mortality Weekly Report 1999; 48 : 7-25.

14. Batista LE. Masculinidade, raça/cor e saúde. Ciên Saúde Colet 2005; 10(1):71-80.

15. OMS. Envelhecimento ativo: uma política de saúde. Brasília: Organização Pan-Americana da Saúde; 2005.

16. Organização Panamericana da Saúde. Doenças crônico-degenerativas e obesidade: estratégia mundial sobre alimentação saudável, atividade física e saúde. Brasília; 2003.

17. Brasil. Ministério da Saúde. Evolução da mortalidade no Brasil. Brasília: Secretaria de Vigilância em Saúde; 2004. p.85-133.

18. Laurenti $\mathrm{R}$, et al. Perfil epidemiológico da saúde masculina na Região das Américas: uma contribuição para o enfoque de gênero. São Paulo: Centro Colaborador da OMS para a Classificação de Doenças em Português; 1998.

19. Pinheiro RS, et al. Gênero, morbidade, acesso e utilização de serviços de saúde no Brasil. Ciên Saúde Colet 2002; 7:687-707.

20. Travassos C, Viacava F. Acesso e uso de serviços de saúde em idosos residentes em áreas rurais, Brasil, 1998 e 2003. Cad Saúde Pública 2007; 23(10):2490-502.

21. Brasil. Ministério da Saúde. Política Nacional de Atenção Integral à saúde do Homem. Princípios e Diretrizes. Brasília: Secretaria de atenção à saúde; 2008.

22. Gomes R, Nascimento EF, Araújo FC. Por que os homens buscam menos os serviços de saúde do que as mulheres? As explicações de homens com baixa escolaridade e homens com ensino superior. Cad Saúde Pública 2007; 23(3):565-74.

23. Brasil. Ministério da Saúde. Secretaria de Vigilância em Saúde. Secretaria de Gestão Estratégica e Participativa. Vigitel Brasil 2008: vigilância de fatores de risco e proteção para doenças crônicas por inquérito telefônico / Ministério da Saúde, Secretaria de Vigilância em Saúde, Secretaria de Gestão Estratégica e Participativa. - Brasília: Ministério da Saúde, 
2009. acesso em: 15 set. 2009. Disponível em: http://www.saude.gov.br/bvs.

24. Alves LC, et al. Influência das doenças crônicas na capacidade funcional dos idosos do município de São Paulo, Brasil. Cad Saúde Pública 2007; 23(8):1924-30.

25. Ford ES, Giles WH, Dietz WH. Prevalence of the metabolic syndrome among US adults: findings from the third National Health and
Nutrition Examination Survey. JAMA 2002;287:356-9.

26. Toscano CM. As campanhas nacionais para detecção das doenças crônicas nãotransmissíveis: diabetes e hipertensão arterial. Ciên Saúde Colet 2004; 9: 885-95.

27. Matijascic M, Dias MPP. Terceira Idade e esperança de vida: o Brasil no cenário internacional. Rio de Janeiro: IPEA; 2008.

Recebido: $11 / 3 / 2009$

Revisado: 14/10/2009

Aprovado: 28/1/2010 
